\title{
EVALUACIÓN DEL CARTÍLAGO ARTICULAR CON RESONANCIA MAGNÉTICA
}

\author{
Dr. Gonzalo Delgado P. \\ Departamento de Imágenes, Clínica Alemana Santiago. \\ Servicio de Radiología, Tomografía Computada y Ultrasonido, Hospital del Trabajador, Santiago. \\ Servicio de Resonancia Magnética, Clínica Avansalud Providencia, Santiago. \\ e-mail: gdelgado@alemana.cl
}

\section{ASSESSMENT OF ARTICULAR CARTILAGE USING MAGNETIC RESONANCE IMAGING}

\section{ABSTRACT}

Articular cartilage injuries is a common event and imaging technique has become increasingly important in diagnosing them. Magnetic Resonance Imaging (MRI) is the imaging method of choice for the evaluation of chondral lesions. This article reviews the usefulness of this method compared to conventional as well as to advanced quantitative studies, thus enabling evaluation of initial cartilage alterations before being evident in normal MRI sequences.

Keywords: Articular cartilage, Chondral lesions, Magnetic Resonance Imaging.

\section{RESUMEN}

Las lesiones del cartílago articular son frecuentes y su diagnóstico por imagen cada día más importante. La resonancia magnética es el método de diagnóstico por imagen de elección para la evaluación de lesiones condrales. Este artículo revisa la utilidad de este método, en relación a estudios convencionales así como también estudios avanzados, cuantitativos, que permiten evaluar alteraciones condrales iniciales antes de ser evidentes en secuencias habituales de resonancia magnética.

Palabras clave: Cartílago articular, Lesiones condrales, Resonancia magnética.

\section{INTRODUCCIÓN}

Las lesiones de los cartílagos articulares son frecuentes en las distintas articulaciones y sus etiologías multifactoriales, incluyendo causas traumáticas, artropatías inflamatorias, infecciosas (artritis séptica) y causas degenerativas. Las lesiones de origen degenerativo son las más frecuentes, siendo un problema de salud pública importante por el alto costo económico y social que representan los gastos directos o indirectos en relación al tratamiento y ausencia laboral. Se calcula que aproximadamente el $75 \%$ de la población mayor de 75 años presentan artrosis ${ }^{(1)}$.

El cartílago articular o cartílago hialino es de vital importancia en las articulaciones de tipo diartrosis (articulaciones con amplio rango de movimiento) y sus funciones principales son disipar y transmitir las fuerzas sobre las superficies articulares, amortiguar las cargas y proveer una superficie de deslizamiento adecuada entre las superficies articulares. Como características principales el cartílago hialino es un tejido avascular (se nutre a través del líquido sinovial), no tiene inervación y no tiene capacidad de regenerarse con el mismo tejido, solo presenta capacidad reparativa limitada con fibrocartílago, el que es de menor resistencia. 
1):
El cartílago articular se compone de (Figura

A. Agua (65-80\%): Está presente en mayor cantidad en las porciones superficiales del cartílago y su contenido aumenta con el proceso de envejecimiento y en las alteraciones degenerativas.

B. Colágeno (10-20\%): El colágeno que predomina es el tipo II (95\%), corresponde a la matriz de sostén del cartílago y provee resistencia a las fuerzas de tensión. El colágeno es el principal componente en el cartílago deshidratado.

C. Proteoglicanos (10-15\%): Son producidos por los condrocitos, siendo sus subunidades, los glicosaminoglicanos (GAG). Proveen resistencia a las fuerzas de compresión y tienen resistencia elástica.

D. Condrocitos (5\%): Corresponden a la parte celular del cartílago y son los encargados de producir los proteoglicanos, el colágeno, las proteínas y algunas enzimas.

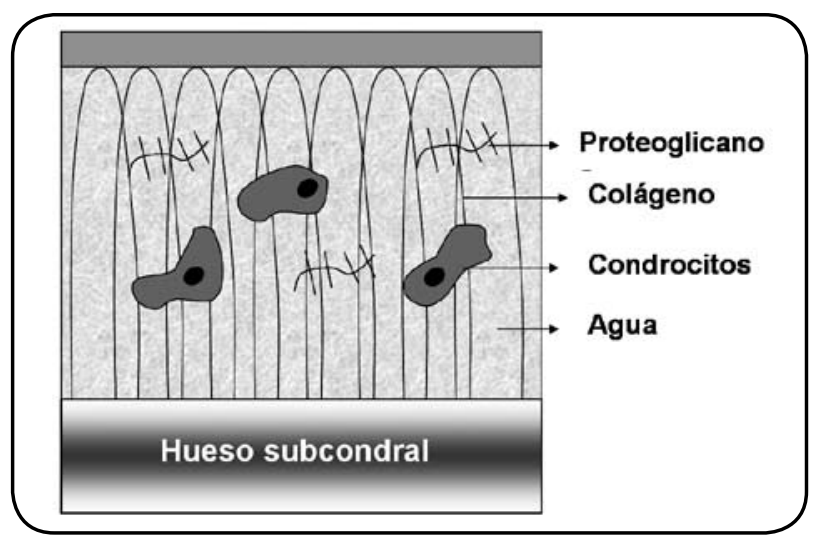

Figura 1. Esquema que muestra los distintos componentes del cartílago articular.

En el cartílago articular se reconocen distintas porciones dependiendo de su profundidad, y la orientación que adquieren las fibras de colágeno (Figura 2). Así, se reconoce una porción superficial que abarca aproximadamente el 10 a $20 \%$ del grosor del cartílago, donde las fibras de colágeno se disponen en forma paralela a la superficie del cartílago. La porción transicional que corresponde al $40-60 \%$, donde las fibras de colágeno tienen una disposición aleatoria. En la porción radial correspondiente al $30 \%$ aproximadamente, las fibras de colágeno tienen una disposición perpendicular a la superficie y es la porción donde el entrelazado de colágeno es más compacto. Por último la lámina calcificada corresponde a la zona donde el cartílago de fusiona a la cortical articular ósea.

Los procesos de envejecimiento y degeneración del cartílago articular se asocian a pérdida de la capacidad reproductiva de los condrocitos, disminución de los proteoglicanos, el cartílago se hace más rígido

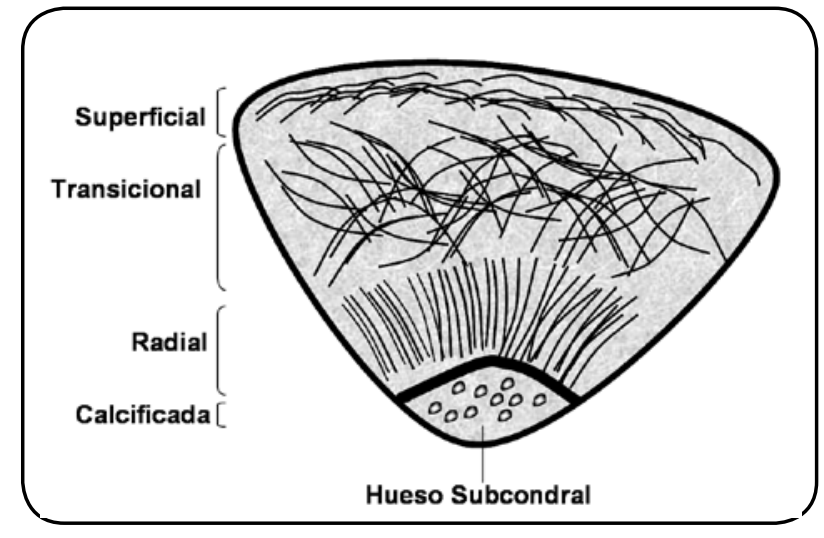

Figura 2. Esquema de las distintas porciones del cartílago articular, dependiendo de la orientación de las fibras de colágeno.

y aumenta su contenido de agua. Estas alteraciones en conjunto determinan una pérdida de las características y funciones del cartílago haciéndolo menos resistente y proclive a lesionarse con ulceraciones $y$ fisuras.

Se pueden encontrar varias clasificaciones de lesiones condrales tanto del punto de vista imaginológico como artoscópico. En este aspecto es importante la comunicación con los clínicos, especialmente traumatólogos, debiendo los radiólogos usar clasificaciones conocidas y usadas por los clínicos que interactúan con nosotros y si es necesario adecuar las clasificaciones fundamentalmente artroscópicas a nuestros informes imaginológicos. Una de las clasificaciones más utilizada por los clínicos dedicados al tema de las lesiones condrales es la clasificación de la ICRS (international Cartilage Repair Society) (Cuadro 1). Esta clasificación artroscópica es fácil de extrapolar a nuestros estudios de imagen pues se basa fundamentalmente en la profundidad de la lesión.

Cuadro 1. Clasificación ICRS (International Cartilage Repair Society) de las lesiones condrales

$0 \quad$ Normal

1 A Fibrilación superficial o reblandecimiento

B Fisuras superficiales y laceraciones

2 Defecto $<50 \%$

3 A Defecto $>50 \%$ sin alcanzar lámina calcificada

B Defecto $>50 \%$ alcanza lámina calcificada

4 A Defecto total con compromiso de placa subcondral

B Defecto total compromiso profundo a placa subcondral 
La resonancia magnética $(\mathrm{RM})$ es el método de elección para evaluar lesiones del cartílago articular por su carácter no invasivo, alto contraste y capacidad multiplanar.

El rendimiento de la RM en la detección de lesiones condrales dependerá del equipo que se use, siendo ideal para la evaluación de lesiones de cartílagos articulares contar con resonadores de alto campo de 1,5 ó 3 Tesla. La sensibilidad de la RM es directamente proporcional a la magnitud en cuanto a superficie condral comprometida y profundidad de la lesión ${ }^{(2)}$. Por otro lado los cartílagos de mayor grosor como los de la rodilla son más fáciles de evaluar que lo cartílagos de aquellas articulaciones más pequeñas. Es importante, siempre y en todas los exámenes de $\mathrm{RM}$ articular buscar en forma dirigida las lesiones condrales, pues es habitual que al revisar retrospectivamente y comparar con hallazgos quirúrgicos se vean lesiones que pasaron desapercibidas en la primera lectura. Las características de las lesiones condrales, especialmente cuando son focales y únicas, que debemos precisar en el informe de la RM se resumen en el cuadro 2.

Cuadro 2. Características importantes de informar en una lesión condral focal.

1. Extensión en superficie midiendo extensión AP y transversal.

2. Profundidad de la lesión (porcentaje del espesor del cartílago comprometido).

3. Ubicación en la superficie articular (compromiso de zona de carga).

4. Alteraciones del hueso subcondral (edema, quistes)

5. Cuerpos condrales u osteocondrales intraarticulares.

Mucho se ha publicado respecto a las secuencias más útiles para evaluación de cartílagos articulares. Las secuencias con buen contraste entre el cartílago y líquido y buen contraste entre cartílago y hueso subcondral son las adecuadas para la evaluación de patología condral. Las secuencias que mejor cumplen con estas condiciones de contraste son especialmente FSE DP con supresión de grasa y SPGR T1 con saturación de grasa. En FSE DP con saturación de grasa el cartílago se ve de señal intermedia, el líquido de alta señal y el hueso subcondral de baja señal (Figura 3). En SPGR T1 con supresión de grasa el cartílago se ve de alta señal, y el líquido de baja señal al igual que el hueso subcondral (Figura 4). Esta última secuencia efectuada con técnica 3D permite realizar cortes finos.

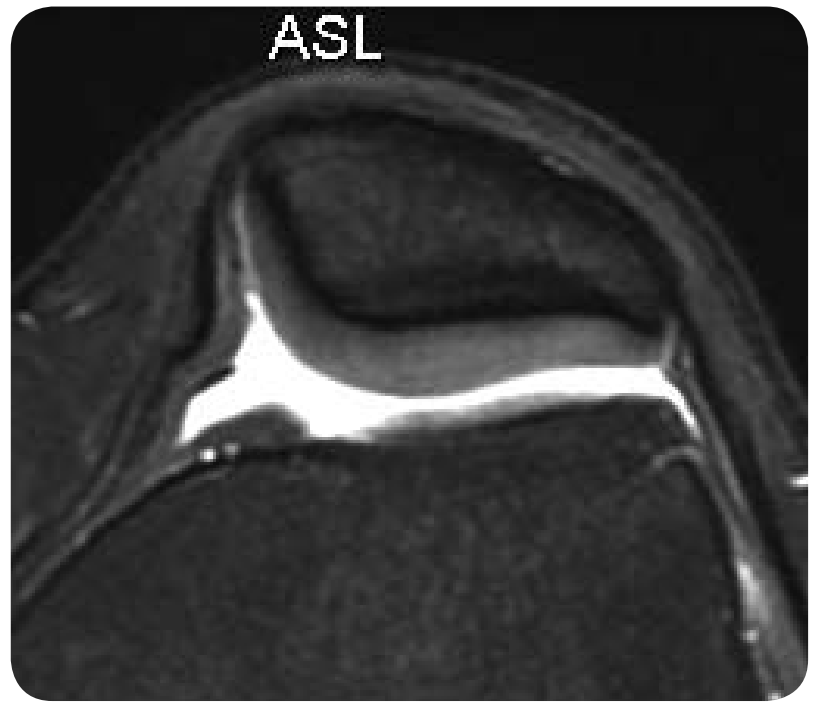

Figura 3. Corte axial potenciado en densidad protón con supresión de la grasa mostrando el cartílago rotuliano. El cartílago es de señal intermedia contrastando con el líquido articular de alta señal.

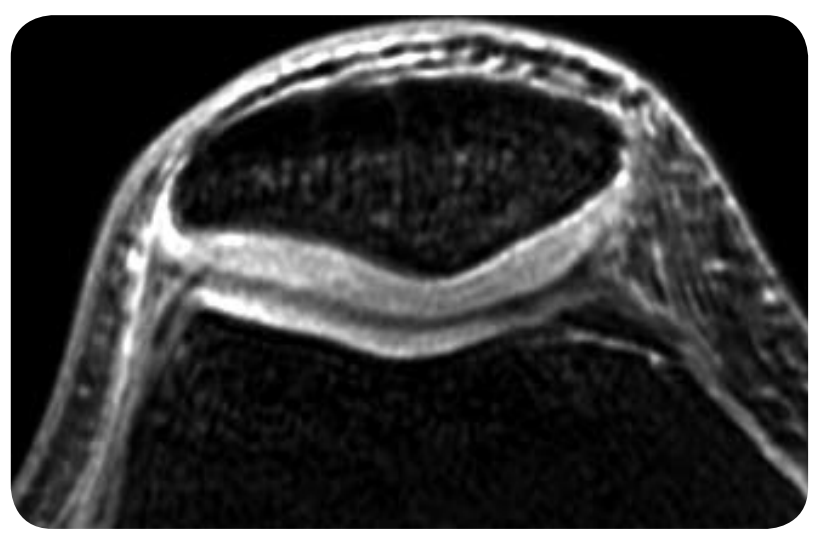

Figura 4. Corte axial en secuencia gradiente SPGR potenciado en T1 mostrando el cartílago rotuliano y de tróclea femoral. El cartílago es de alta señal contrastando con el líquido articular de baja señal.

Las secuencias T2 presentan buen contraste entre cartílago (baja señal) y líquido (alta señal), sin embargo el contraste entre cartílago y hueso cortical subcondral no es adecuada puesto que ambos presentan baja señal. Aunque esta secuencia no es muy sensible a alteraciones sutiles, es importante en las lesiones condrales traumáticas.

Existe bastante consenso en cuanto a que las secuencias más útiles serían las secuencias FSE DP con supresión de la grasa y SPGR T1 con saturación de grasa $^{(3)}$. Estas secuencias tienen buen contraste y son sensibles a las alteraciones patológicas del cartílago articular lo que las hace las mejores secuencias.

Como se mencionó previamente es importante buscar dirigidamente las lesiones de cartílago independiente del protocolo usado para el estudio. 
Las lesiones condrales de origen traumáticas son típicamente solitarias, de contornos bien definidos, y con frecuencia comprometen todo el espesor del cartílago (Figura 5) y se pueden asociar a cuerpos osteocondrales o condrales intraarticulares los que pueden provocar bloqueo articular. Las lesiones de origen degenerativas se inician con alteraciones bioquímicas intra-sustancia, siguiendo con fibrilación, fisuras, ulceraciones de espesor total y por último pérdida de cartílago de espesor total. Habitualmente las lesiones degenerativas son de contornos irregulares, y frecuentemente comprometen más de un sector de las superficies articulares con lesiones de espesor variable y dependiendo de la etapa del compromiso se pueden observar alteraciones secundarias de artrosis como osteofitos marginales, quistes y alteraciones de señal del hueso subcondral (Figura 6).
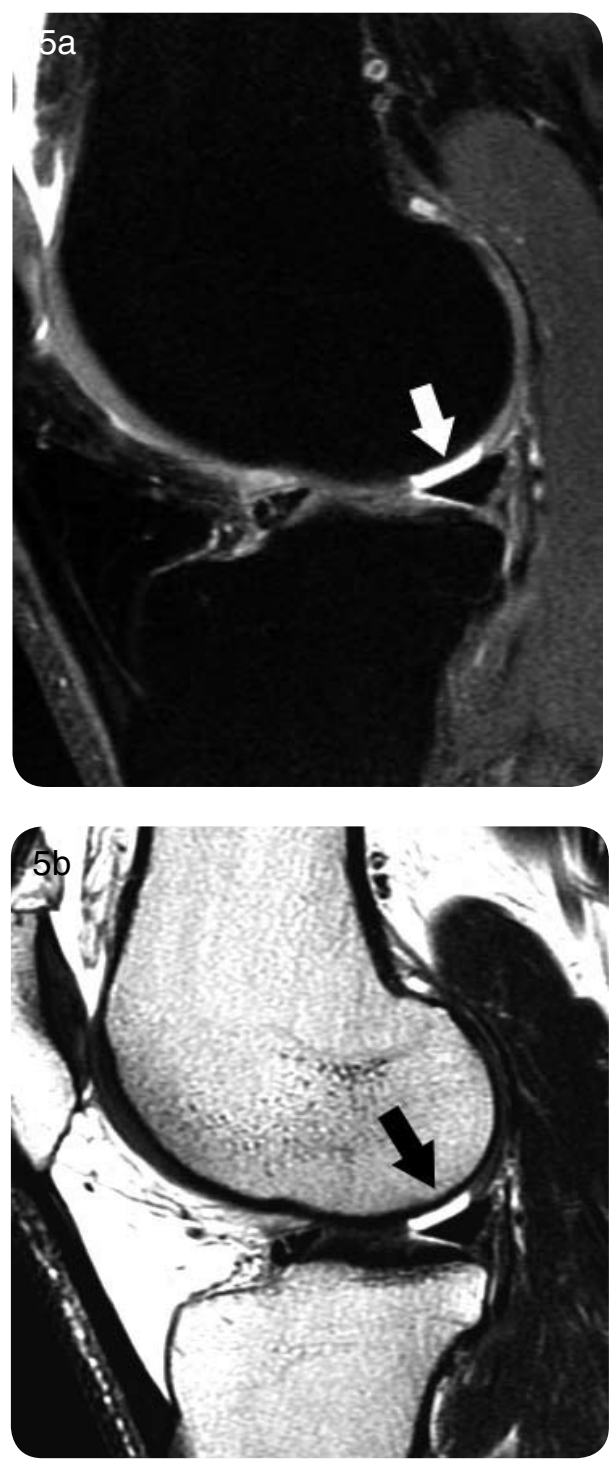

Figura 5. Cortes sagitales del compartimiento femorotibial externo de la rodilla potenciado en DP con saturación de grasa (a) y T2 (b). Se puede ver una lesión condral focal de espesor total, de origen traumático.

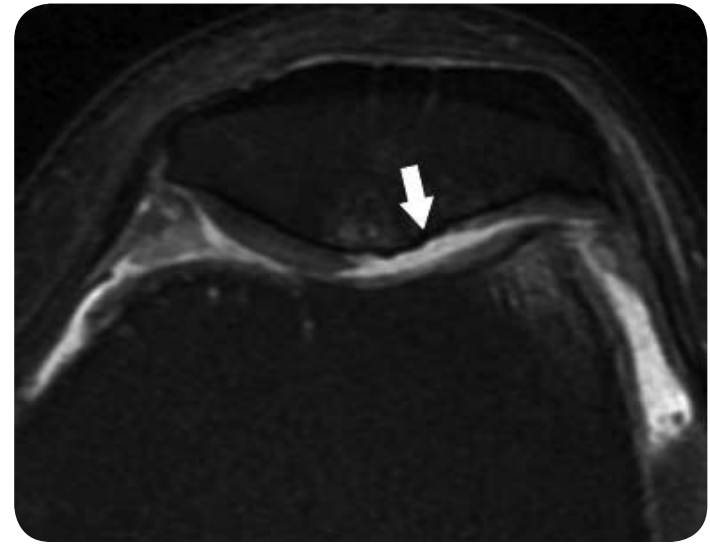

Figura 6. Corte axial potenciado en DP con saturación de grasa mostrando condropatía degenerativa avanzada de la rótula.

La RM es importante en la evaluación de lesiones condrales tratadas en forma quirúrgica. En este sentido las dos técnicas quirúrgicas más frecuentemente usadas son la microfractura y los injertos osteocondrales autólogos (mosaicoplastía, OATS). La microfractura consiste en realizar perforaciones en la zona de lesión condral lo que produce una zona cruenta de hueso subcondral con formación de grupo de células mesenquimales indiferenciadas de la médula ósea que formarán fibrocartílago reparativo, el que es de menor resistencia que el cartílago hialino. Los injertos osteocondrales autólogos fundamentalmente usados en lesiones condrales en superficies de carga de la rodilla consiste en sacar un fragmento osteocondral en zona de no carga (habitualmente tróclea femoral) para colocarlos en la zona de lesión condral original. Este procedimiento aunque mas demandante técnicamente tiene la ventaja de reparar la lesión con cartílago hialino.

\section{ESTUDIOS AVANZADOS DE RM}

Se han desarrollado métodos especiales de RM para evaluación de cartílagos articulares. La mayoría de ellos son estudios que permanecen más bien en investigación sin aplicación clínica importante, siendo la excepción el estudio mapa T2 el que se ha desarrollado más en la práctica diaria y será el revisado con más detalle.

\section{MAPA T2}

El estudio mapa T2 del cartílago articular es un método cuantitativo de evaluación de la estructura interna del cartílago. Con esta técnica es posible medir el tiempo de relajación T2 del cartílago.

Los tiempos de relajación T2 en el cartílago normal son menores en las capas más profundas donde el entrelazado de fibras de cartílago es más compacto y hay menos cantidad de agua. Los tiempos de relajación T2 aumentan hacia las porciones más superficiales del cartílago. 
Este método se basa fundamentalmente en que las alteraciones degenerativas producen desorganización de la matriz de colágeno, la que se hace más laxa permitiendo mayor contenido de protones de $\mathrm{H}_{2} \mathrm{O}$ los que además están más libres, lo que produce aumento de los valores de relajación T2 por sobre niveles normales.

Con el programa adecuado, en la estación de trabajo es posible medir el tiempo de relajación T2 en milisegundos, situando el área de interés donde se estime necesario, lo que permite cuantificar objetivamente las alteraciones. Además esto se puede representar morfológicamente en imagen de colores usando escala predefinida para hacerlo visualmente detectable (Figura 7). Como este no es un estudio morfológico sino más bien cuantitativo su mayor utilidad es detectar alteraciones intra-sustancia iniciales del cartílago previo a las ulceraciones o fisuras de su superficie (Figura 8 ). Esta capacidad hace que este método pueda evaluar la evolución de alteraciones condrales incipientes después de tratamientos farmacológicos o de otra naturaleza. De las técnicas más avanzadas para evaluación de cartílago esta es la que se está usando más en la práctica clínica ${ }^{(4,5,6)}$.

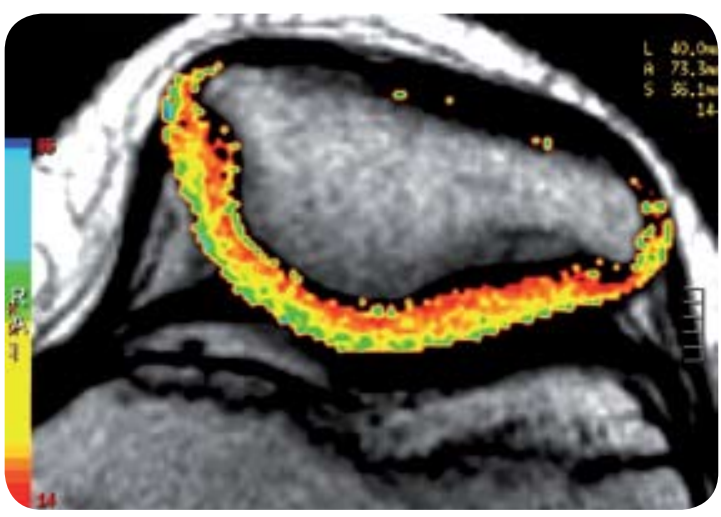

Figura 7. Mapa T2 con escala de colores de cartílago rotuliano normal donde la parte más profunda muestra coloración roja indicando niveles bajos de tiempos de relajación $\mathrm{T} 2$, la parte central coloración amarilla y la zona más superficial coloración verde indicando niveles más altos de T2.

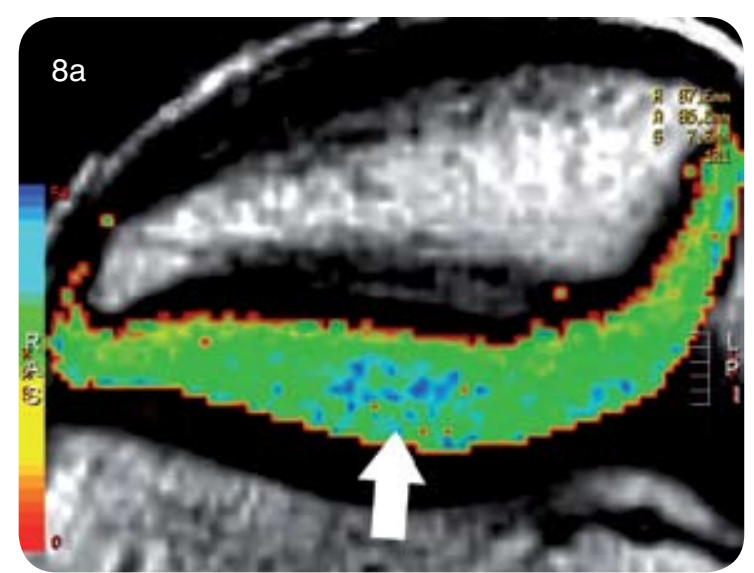

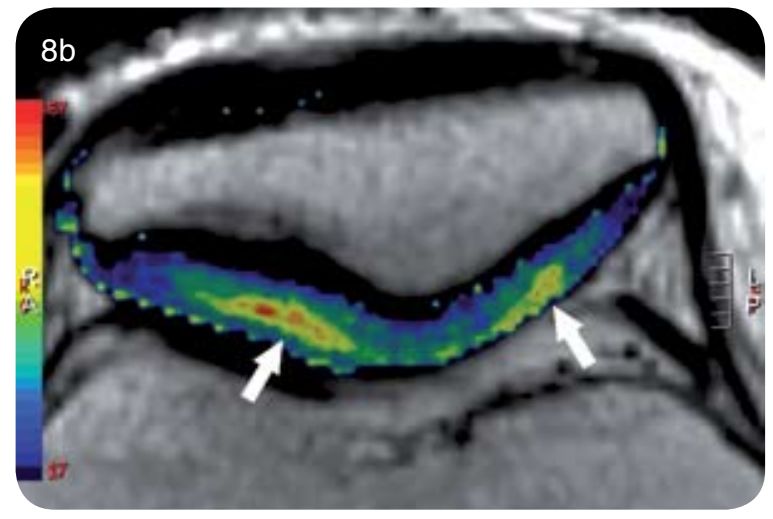

Figura 8. Mapa T2 color en dos distintos pacientes mostrando zonas alteradas (flechas) con aumento de niveles T2 en el espesor del cartílago articular.

\section{REFUERZO RETARDADO CON GADOLINIO}

Este método consiste en inyectar por vía intravenosa gadolinio iónico que tiene cargas negativas, y efectuar movilidad activa y ejercicio de la articulación en estudio lo que permitiría paso de contraste hacia el líquido sinovial. Este método permite evaluar la concentración de proteoglicanos en el cartílago $\operatorname{articular}^{(7)}$.

Este estudio se basa en las cargas negativas que tienen los glicosaminoglicanos, que son las subunidades de los proteoglicanos. Se conoce que con los procesos degenerativos y de envejecimiento del cartílago articular disminuye la cantidad de proteoglicanos. Si existe cantidad normal de glicosaminoglicanos (carga (-)) el contraste (carga $(-))$ será repelido y no penetrará por difusión al cartílago. Cuando la cantidad de glicosaminoglicanos está disminuida permite que el contraste penetre e impregne el cartílago en las zonas alteradas. Esta mayor captación se puede representar en imagen color.

Dentro de otros métodos avanzados de estudio y fundamentalmente usados en investigación más que en aplicación clínica, podemos mencionar técnicas específicas como proyección reconstrucción de tiempo corto de eco, espectroscopía de cartílago entre otros.

En resumen, el cartílago articular es un tejido altamente resistente, sin embargo, sus lesiones son frecuentes y la RM es el método de imagen de elección para su evaluación. Para esto son útiles las secuencias convencionales, existiendo también algunas técnicas especializadas de RM que pueden permitir evaluación más objetiva, cuantitativa, de las alteraciones condrales degenerativas incipientes. Varias de estas técnicas especiales están todavía en proceso de desarrollo e investigación y aun no han sido aplicadas a la práctica clínica. Excepción a esto es el estudio mapa T2 de cartílago el que está siendo usado más rutinariamente. 


\section{BIBLIOGRAFÍA}

1. Lawrence RC, Hochberg MC, Kelsey JL, McDuffie FC, Medsger TA Jr, Felts WR, et al. Estimates of the prevalence of selected arthritic and musculoskeletal diseases in the United States: J Rheumatol. 1989; 16(4): 427-41.

2. Figueroa D, Calvo R, Vaisman A, Carrasco M, Moraga $C$, Delgado I. Knee chondral lesions ; Incidente and correlation between Arthroscopic and magnetic resonance findings: Arthroscopy. 2007; 23(3): 312-5.

3. Recht M, Goodwin D, Winalski C, White L. MRI of articular cartilage: Revisiting current status and future directions. AJR 2005; 185(4): 899-914.

4. Dunn T, Lu Y, Jin H, Ries M, Majumdar S. T2 relaxation time of cartilage at MR imaging: comparison with se- verity of knee osteoarthritis. Radiology. 2004; 232(2): 592-8.

5. Mosher T, Dardzinski B, Smith M. Human articular cartilage influence of aging and early symptomatic degeneration on the spatial variation of T2-Preliminary findings at 3T. Radiology. 2000; 214(1): 259-66.

6. Watrin A, Ruaud JP, Olivier PT, Guingamp NC, Gonord PD, Netter PA, et al. T2 mapping of rat patellar cartilage. Radiology 2001; 219(2): 395-402.

7. Young A, Stanwell P, Williams A, Rohrsheim J, Parker D, Giuffre B, et al. Glycosaminoglycan content of knee cartilage following posterior cruciate ligament rupture demonstrated by delayed gadolinium-enhanced magnetic resonance imaging of cartilage (dGEMRIC). A case report. J Bone Joint Surg Am. 2005; 87(12): 2763-7. 